\title{
A Screw Syzygy with Applications to Robot Singularity Computation
}

\author{
J.M. Selig and Peter Donelan
}

Faculty of Business, Computing and Information Management, London South Bank University, U.K.

and

School of Mathematics, Statistics and Computer Science

Victoria University of Wellington, New Zealand. 


\section{What is a Syzygy}

A syzygy is a relation between invariants.

The invariants we have in mind here are rigid invariants of screws. Consider a pair of screws (twists),

$$
\mathbf{s}_{1}=\left(\begin{array}{l}
\boldsymbol{\omega}_{1} \\
\mathbf{v}_{1}
\end{array}\right) \quad \text { and } \quad \mathbf{s}_{2}=\left(\begin{array}{c}
\boldsymbol{\omega}_{2} \\
\mathbf{v}_{2}
\end{array}\right)
$$

where $\boldsymbol{\omega}_{1}, \boldsymbol{\omega}_{2}, \mathbf{v}_{1}$ and $\mathbf{v}_{2}$ are 3 -vectors, then $\ldots$ 
... the sort of screw invariants we have in mind are,

$$
\mathbf{s}_{1}^{T} Q_{0} \mathbf{s}_{2}=\left(\omega_{1}^{T}, \mathbf{v}_{1}^{T}\right)\left(\begin{array}{ll}
0 & l \\
l & 0
\end{array}\right)\left(\begin{array}{l}
\omega_{2} \\
\mathbf{v}_{2}
\end{array}\right)=\omega_{1} \cdot \mathbf{v}_{2}+\mathbf{v}_{1} \cdot \omega_{2}
$$

This is the reciprocal product of screws and is well known to be invariant under rigid transformations of both screws. 


\section{An SO(3) Syzygy}

An example of a syzygy for the group of rotations is given by the following. Let $\mathbf{a}_{1}, \mathbf{a}_{2}$ and $\mathbf{a}_{3}$ be any three 3-vectors, then the following relation holds,

$$
\left(\mathbf{a}_{1} \cdot\left(\mathbf{a}_{2} \times \mathbf{a}_{3}\right)\right)^{2}=\operatorname{det}\left(\begin{array}{lll}
\mathbf{a}_{1} \cdot \mathbf{a}_{1} & \mathbf{a}_{1} \cdot \mathbf{a}_{2} & \mathbf{a}_{1} \cdot \mathbf{a}_{3} \\
\mathbf{a}_{2} \cdot \mathbf{a}_{1} & \mathbf{a}_{2} \cdot \mathbf{a}_{2} & \mathbf{a}_{2} \cdot \mathbf{a}_{3} \\
\mathbf{a}_{3} \cdot \mathbf{a}_{1} & \mathbf{a}_{3} \cdot \mathbf{a}_{2} & \mathbf{a}_{3} \cdot \mathbf{a}_{3}
\end{array}\right)
$$

According to Weyl all invariants of the standard representation of $S O$ (3) (i.e. its action on vectors in $\mathbb{R}^{3}$ ) are generated by the scalar product of pairs of vectors and the scalar triple product of triples of vectors. And all relations between the invariants can be derived from the above relation. 


\section{An SE(3) Syzygy}

We believe this relation to be new. Let $\mathbf{s}_{1}, \ldots, \mathbf{s}_{6}$ be six screws, consider the matrix with these screws as columns,

$$
J=\left(\mathbf{s}_{1}\left|\mathbf{s}_{2}\right| \mathbf{s}_{3}\left|\mathbf{s}_{4}\right| \mathbf{s}_{5} \mid \mathbf{s}_{6}\right)
$$

Now we have that,

$$
\operatorname{det}(J)^{2}=-\operatorname{det}\left(\mathbf{s}_{i}^{T} Q_{0} \mathbf{s}_{j}\right)
$$

That is, the square of the determinant of the Jacobian is minus the determinant of the matrix of reciprocal products. 


\section{Proof}

The matrix of reciprocal products is clearly given by,

$$
J^{T} Q_{0} J=\left(\begin{array}{lllllll}
\mathbf{s}_{1}^{T} Q_{0} \mathbf{s}_{1} & \mathbf{s}_{1}^{T} Q_{0} \mathbf{s}_{2} & \mathbf{s}_{1}^{T} Q_{0} \mathbf{s}_{3} & \mathbf{s}_{1}^{T} Q_{0} \mathbf{s}_{4} & \mathbf{s}_{1}^{T} Q_{0} \mathbf{s}_{5} & \mathbf{s}_{1}^{T} Q_{0} \mathbf{s}_{6} \\
\mathbf{s}_{2}^{T} Q_{0} \mathbf{s}_{1} & \mathbf{s}_{2}^{T} Q_{0} \mathbf{s}_{2} & \mathbf{s}_{2}^{T} Q_{0} \mathbf{s}_{3} & \mathbf{s}_{2}^{T} Q_{0} \mathbf{s}_{4} & \mathbf{s}_{2}^{T} Q_{0} \mathbf{s}_{5} & \mathbf{s}_{2}^{T} Q_{0} \mathbf{s}_{6} \\
\mathbf{s}_{3}^{T} Q_{0} \mathbf{s}_{1} & \mathbf{s}_{3}^{T} Q_{0} \mathbf{s}_{2} & \mathbf{s}_{3}^{T} Q_{0} \mathbf{s}_{3} & \mathbf{s}_{3}^{T} Q_{0} \mathbf{s}_{4} & \mathbf{s}_{3}^{T} Q_{0} \mathbf{s}_{5} & \mathbf{s}_{3}^{T} Q_{0} \mathbf{s}_{6} \\
\mathbf{s}_{4}^{T} Q_{0} \mathbf{s}_{1} & \mathbf{s}_{4}^{T} Q_{0} \mathbf{s}_{2} & \mathbf{s}_{4}^{T} Q_{0} \mathbf{s}_{3} & \mathbf{s}_{4}^{T} Q_{0} \mathbf{s}_{4} & \mathbf{s}_{4}^{T} Q_{0} \mathbf{s}_{5} & \mathbf{s}_{4}^{T} Q_{0} \mathbf{s}_{6} \\
\mathbf{s}_{5}^{T} Q_{0} \mathbf{s}_{1} & \mathbf{s}_{5}^{T} Q_{0} \mathbf{s}_{2} & \mathbf{s}_{5}^{T} Q_{0} \mathbf{s}_{3} & \mathbf{s}_{5}^{T} Q_{0} \mathbf{s}_{4} & \mathbf{s}_{5}^{T} Q_{0} \mathbf{s}_{5} & \mathbf{s}_{5}^{T} Q_{0} \mathbf{s}_{6} \\
\mathbf{s}_{6}^{T} Q_{0} \mathbf{s}_{1} & \mathbf{s}_{6}^{T} Q_{0} \mathbf{s}_{2} & \mathbf{s}_{6}^{T} Q_{0} \mathbf{s}_{3} & \mathbf{s}_{6}^{T} Q_{0} \mathbf{s}_{4} & \mathbf{s}_{6}^{T} Q_{0} \mathbf{s}_{5} & \mathbf{s}_{6}^{T} Q_{0} \mathbf{s}_{6}
\end{array}\right)
$$

The result then comes from taking determinants of both sides of this relation and noting that $\operatorname{det}\left(Q_{0}\right)=-1$. 


\section{Wrist Partitioned Serial Manipulator}

Joint screws of a serial robot give columns of the Jacobian. If the robot has a $3 \mathrm{R}$ wrist, last three joints screws are lines meeting at a point. This means last three joint screws are mutually reciprocal, the $3 \times 3$ bottom right corner of the matrix of reciprocal products vanishes. So,

$$
\operatorname{det}(J)= \pm \operatorname{det}\left(\begin{array}{lll}
\mathbf{s}_{1}^{T} Q_{0} \mathbf{s}_{4} & \mathbf{s}_{1}^{T} Q_{0} \mathbf{s}_{5} & \mathbf{s}_{1}^{T} Q_{0} \mathbf{s}_{6} \\
\mathbf{s}_{2}^{T} Q_{0} \mathbf{s}_{4} & \mathbf{s}_{2}^{T} Q_{0} \mathbf{s}_{5} & \mathbf{s}_{2}^{T} Q_{0} \mathbf{s}_{6} \\
\mathbf{s}_{3}^{T} Q_{0} \mathbf{s}_{4} & \mathbf{s}_{3}^{T} Q_{0} \mathbf{s}_{5} & \mathbf{s}_{3}^{T} Q_{0} \mathbf{s}_{6}
\end{array}\right)
$$

If only interested in singularities then sign ambiguity not important. 


\section{Singularities}

Setting this $3 \times 3$ determinant to zero means that the columns (or rows) of the matrix must be linearly dependent.

Column dependence can be expressed by the equations

$$
\begin{aligned}
& \lambda \mathbf{s}_{1}^{T} Q_{0} \mathbf{s}_{4}+\mu \mathbf{s}_{1}^{T} Q_{0} \mathbf{s}_{5}+\nu \mathbf{s}_{1}^{T} Q_{0} \mathbf{s}_{6}=0 \\
& \lambda \mathbf{s}_{2}^{T} Q_{0} \mathbf{s}_{4}+\mu \mathbf{s}_{2}^{T} Q_{0} \mathbf{s}_{5}+\nu \mathbf{s}_{2}^{T} Q_{0} \mathbf{s}_{6}=0 \\
& \lambda \mathbf{s}_{3}^{T} Q_{0} \mathbf{s}_{4}+\mu \mathbf{s}_{3}^{T} Q_{0} \mathbf{s}_{5}+\nu \mathbf{s}_{3}^{T} Q_{0} \mathbf{s}_{6}=0
\end{aligned}
$$

for some non-zero constants $\lambda, \mu$ and $\nu$. 


\section{Wrist Singularity}

These equations can be solved in two ways. First, if last three joints are linearly dependent,

$$
\lambda \mathbf{s}_{4}+\mu \mathbf{s}_{5}+\nu \mathbf{s}_{6}=\mathbf{0}
$$

for some $\lambda, \mu, \nu$, then equations satisfied.

Occurs if and only if the three screw axes $\mathbf{s}_{4}, \mathbf{s}_{5}$ and $\mathbf{s}_{6}$ are coplanar and constitutes a wrist singularity. 


\section{Shoulder Singularity}

Likewise, if the rows of the matrix are linearly dependent then for some $\alpha, \beta, \gamma$ not all zero

$$
\begin{aligned}
\alpha \mathbf{s}_{1}^{T} Q_{0} \mathbf{s}_{4}+\beta \mathbf{s}_{2}^{T} Q_{0} \mathbf{s}_{4}+\gamma \mathbf{s}_{3}^{T} Q_{0} \mathbf{s}_{4}=0 \\
\alpha \mathbf{s}_{1}^{T} Q_{0} \mathbf{s}_{5}+\beta \mathbf{s}_{2}^{T} Q_{0} \mathbf{s}_{5}+\gamma \mathbf{s}_{3}^{T} Q_{0} \mathbf{s}_{5}=0 \\
\alpha \mathbf{s}_{1}^{T} Q_{0} \mathbf{s}_{6}+\beta \mathbf{s}_{2}^{T} Q_{0} \mathbf{s}_{6}+\gamma \mathbf{s}_{3}^{T} Q_{0} \mathbf{s}_{6}=0
\end{aligned}
$$

and the same reasoning leads to a shoulder singularity of the first three joints when they fail to span a 3-dimensional subspace. Whether or not such a singularity can occur depends on the screw systems determined by the first three joints, i.e. on the design parameters of the shoulder. 


\section{The Other Singularities}

The second kind of solution most easily derived from previous equations. Satisfied if and only if there is a screw $\alpha \mathbf{s}_{1}+\beta \mathbf{s}_{2}+\gamma \mathbf{s}_{3}$ reciprocal to each of $\mathbf{s}_{4}, \mathbf{s}_{5}$ and $\mathbf{s}_{6}$, hence to all screws in the screw system $\mathcal{S}$ spanned by the last three joints.

Since their axes intersect in the wrist centre, $\mathcal{S}$ is a type IIA $(p=0)$ 3-system, which is self-reciprocal.

Hence for singularity the screw $\alpha \mathbf{s}_{1}+\beta \mathbf{s}_{2}+\gamma \mathbf{s}_{3}$ lies in $\mathcal{S}$ so it must also be a line through the wrist centre. 


\section{The Other Singularities, continued}

In general, set of lines in the 3-system formed by $\mathbf{s}_{1}, \mathbf{s}_{2}$ and $\mathbf{s}_{3}$ form a regulus of a hyperboloid. If any of these lines passes through the wrist centre then the configuration of the robot is singular.

In some cases only possibilities are when the wrist centre lies on the first or second joint axis. 


\section{Other Situations}

Some less general situations. Consider the PUMA manipulator: the first and second joint axes meet and the second and third joint axes are parallel.

Lines in this 3-system comprise, (i) the lines in 3-space parallel to and coplanar with, the second and third joint axes, and (ii) the set of lines coplanar with the first and second joint axes meeting their common point.

So the PUMA has singularities if its wrist centre lies in either the plane containing the first and second joints or the plane containing the second and third joints. 


\section{Gough-Stewart platforms}

For Gough-Stewart platforms well known that rows of the Jacobian matrix found from the lines joining the passive spherical joints. If $\mathbf{s}_{i}$ is the unit line joining the passive spherical joints on the $i$ th leg the corresponding row in the Jacobian matrix is $\mathbf{s}_{i}^{T} Q_{0}$. Writing $K$ for this Jacobian and using $Q_{0}^{2}=I$, the syzygy gives

$$
-\operatorname{det}(K)^{2}=\operatorname{det}\left(\mathbf{s}_{i}^{T} Q_{0} \mathbf{s}_{j}\right)
$$




\section{Gough-Stewart platforms, Jacobians}

Screws $\mathbf{s}_{i}$ are lines: diagonal of the matrix of reciprocal products all zeros.

In the 3-3 design pairs of legs meet at the passive spherical joints alternately on the base and the platform. Just three passive spherical joints on each. So consecutive leg axes are reciprocal. Matrix of reciprocal products has the form,

$$
K Q_{0} K^{T}=\left(\begin{array}{cccccc}
0 & 0 & \mathbf{s}_{1}^{T} Q_{0} \mathbf{s}_{3} & \mathbf{s}_{1}^{T} Q_{0} \mathbf{s}_{4} & \mathbf{s}_{1}^{T} Q_{0} \mathbf{s}_{5} & 0 \\
0 & 0 & 0 & \mathbf{s}_{2}^{T} Q_{0} \mathbf{s}_{4} & \mathbf{s}_{2}^{T} Q_{0} \mathbf{s}_{5} & \mathbf{s}_{2}^{T} Q_{0} \mathbf{s}_{6} \\
\mathbf{s}_{3}^{T} Q_{0} \mathbf{s}_{1} & 0 & 0 & 0 & \mathbf{s}_{3}^{T} Q_{0} \mathbf{s}_{5} & \mathbf{s}_{3}^{T} Q_{0} \mathbf{s}_{6} \\
\mathbf{s}_{4}^{T} Q_{0} \mathbf{s}_{1} & \mathbf{s}_{4}^{T} Q_{0} \mathbf{s}_{2} & 0 & 0 & 0 & \mathbf{s}_{4}^{T} Q_{0} \mathbf{s}_{6} \\
\mathbf{s}_{5}^{T} Q_{0} \mathbf{s}_{1} & \mathbf{s}_{5}^{T} Q_{0} \mathbf{s}_{2} & \mathbf{s}_{5}^{T} Q_{0} \mathbf{s}_{3} & 0 & 0 & 0 \\
0 & \mathbf{s}_{6}^{T} Q_{0} \mathbf{s}_{2} & \mathbf{s}_{6}^{T} Q_{0} \mathbf{s}_{3} & \mathbf{s}_{6}^{T} Q_{0} \mathbf{s}_{4} & 0 & 0
\end{array}\right)
$$

Zero-structure of this matrix renders the determinant relatively easy to compute. 


\section{Conclusions}

- Analysis of singularities of wrist-partitioned robot well known, but this description very clear and simple. 


\section{Conclusions}

- Analysis of singularities of wrist-partitioned robot well known, but this description very clear and simple.

- Can apply singularity analysis to other robots with sub-assembly of three consecutive joints forming a self-reciprocal screw system (but only one or two of these). 


\section{Conclusions}

- Analysis of singularities of wrist-partitioned robot well known, but this description very clear and simple.

- Can apply singularity analysis to other robots with sub-assembly of three consecutive joints forming a self-reciprocal screw system (but only one or two of these).

- Can use above to compute determinants of robot Jacobians quickly. Cholesky decomposition for symmetric matrices known to be twice as fast as standard LU decomposition. 


\section{Conclusions}

- Analysis of singularities of wrist-partitioned robot well known, but this description very clear and simple.

- Can apply singularity analysis to other robots with sub-assembly of three consecutive joints forming a self-reciprocal screw system (but only one or two of these).

- Can use above to compute determinants of robot Jacobians quickly. Cholesky decomposition for symmetric matrices known to be twice as fast as standard LU decomposition.

- Other syzygies can be derived from above, probably useful. 